\section{ESTRUCTURAS DE MADERA LAMINADA}

\author{
Ignacio Seoane Y Oriz de Millajos \\ lugeniaro de Montes. \\ Destatamento de Nareras. Instituro Nacional \\ d. Muestigaciones araras
}

250.5

Le construcción de vigas de madera de gran longutud seremonta más de cuatrocientos años, pero lue a li. nales del siglo pasado cuando se pudo obtener una sección homogénea, sin deshizamento relativo de una lámina con relación a la otra, gracias la genial idea del suizo Otto Mezer que utilizó la cola de caseína pa" ra asegurar de manera intima la unión de las planchas entre s?

De esca idea nació un material, del cual el récord alcanzado no hace más que crecer.

Se denomina madera laminada toda pieza recta o curvada obtenida a partir de piezas menores en forma de tablas o tablillas, encoladas en capas sucesivas en los tres direcciones, de tal foma que las fibras de to das las piezas sean paralelas ente st y a la dirección longirudinal de la pieza, predominando una dimensión con relación a las dos restantes.

El gran auge de este tipo de estructuras se debe principaimente al desarrollo conseguido en la rabricación de las colas, especialmente a partir de la introducción de las colas de resinas sintéticas, que son mucho más ra. sistentes a los factores clináticos, causantes del empleo limitado hasta hace poco de las estructuras laminadas en exteriores.

Entre las ventajas cue presentan las estructuras de ma" dera laminada podemos citar las siguientes:

- Posibilidad de fabricar elementos de sección trans. versal y longiud muy superiores a las que se puen den obrener con una sola pleza de madera ase rrada.

- Calidades de madera inuthizables como elementos astucturales pueden empleare en las estructuras de madera laminada para forma un elemento con las mismas caracteristicas resistentes que cores. ponden a una pieza única.

Como es conocido, una viga sometida a frexión pre. senta en cada sección transvesal una distribución lineấ de rensiones. En las láminas próximas a la fibra neutra, las tensiones son my reducidas, lo que permi- ta emplear maderas de calidad imerior, con lo que se consigue una economia sustancial:

- Posibilidad de construir elementos de sección va. riable, que se adapten con las secciones estricta. mente mecesarias a las soliciraciones acuanares.

- Existe un control muy superior de la calldad de la madera.

muprovechamiento de piezas de madera de peque. ñas dimensiones.

- Consecución de elementos estructurales, decorát. vos por simismos, que no necesisan orácicamente ningún tratamiento posterior ni acabados espe. ciales.

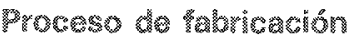

Para melor comprender este tipo de estructuras, recor. daremos brevemente el proceso de su tabricación, que tiende, cada vez más, a una automatzación de ciertes funciones:

- Amecenaje de la madera en parquet.

- Secado de la madera en secadero a fin de obtener la humedad compatible con el tipo de cola emplea" do y ar runción del destino de la estructura.

- Selección y cleshicación de las tablas segun cali. dades.

- Troceado y empalme ce las láminas a fin de realizar las longitudes necesarias a la fabricación.

- Capilado de láminas.

- Encolado de láminas.

- Amado de los elementos encolados.

- Prensado a fin de obtener una presión unitorme. Esta presión es función de la cola elegida y de la especie de madera.

- Estabilización de las vigas a temperatura constante después del cepillado sobre las dos caras.

- Trabajos de acabado entallado pertorado y colocación de los eternentos de unión.

- Tratamiento de la estructura.

- Amacenaje de los elementos acabados.

Asfrabricada y gracias a los controles otectudos en cada lase de fabricación, la madera laminada aponta to das las garantas desda el punto de vista de la calldad y resistencia del material acabado.

\section{Caracteríntcas we las colas y maderas}

La madera laminada, como ya hemos dicho, se compo. ne de dos elementos fundamentales: madera y cola.

El empleo de los direrentes tipos de coles depende de las condiciones de exposición a las que va a estar so. metida la estructura.

En el cuadro siguiente se resumen los tipos de colas empleados según las condiciones de explotación. 


\begin{tabular}{|c|c|c|c|}
\hline $\begin{array}{l}\text { lemperatura } \\
\text { ambiente }\end{array}$ & $\begin{array}{l}\text { Empleo en interior. } \\
\text { Humedad de estabizacion de la } \\
\text { madera }<18 \% \text {. }\end{array}$ & $\begin{array}{l}\text { Emples an amóstera húmeda. } \\
\text { Humedad de estabilizasion de la } \\
\text { madera }>18 \% \text {. }\end{array}$ & $\begin{array}{l}\text { Exposición a la intemperie o atu } \\
\text { mósíta que contiene productos } \\
\text { quimicos. }\end{array}$ \\
\hline elevada (1) & $\begin{array}{l}\text { Resorcina-formol. } \\
\text { Resorcina-fenol-formol. } \\
\text { Caseina. } \\
\text { Melamina-tormol. } \\
\text { Melamina-fenol-formol. }\end{array}$ & 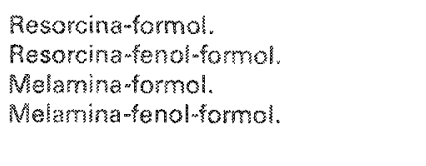 & $\begin{array}{l}\text { Resorcina-yomol. } \\
\text { Resorina - }\end{array}$ \\
\hline nomal(2) & $\begin{array}{l}\text { Resorina-fomol. } \\
\text { Resorcina-fenol-formol. } \\
\text { Urea-Fomol. } \\
\text { Caseina. } \\
\text { Melamina-formol. } \\
\text { Melamina fenol-formol. }\end{array}$ & $\begin{array}{l}\text { Aesorina fomol. } \\
\text { Resorina wenoltormol. } \\
\text { Urea-formol. } \\
\text { Melamina fomol, } \\
\text { Melamina-fenol-formol. }\end{array}$ & $\begin{array}{l}\text { Resorcina-formol: } \\
\text { Resorcinawenolformol. }\end{array}$ \\
\hline
\end{tabular}

(1) La pemperatura ambiente an la proximidad de lo astructura supera los 300 an perodos superioyes a varias hores consteutivis.

(2) La temperatura anoiente en la proximidad de la estructura no supera los 30 en periodos superioras a warias haras consecumas.

En cuanto a las maderas, una propiedad importante que deben observar es su aptitud al encolado.

En la Noma Española, que se encuentra en fase de elaboracion, se agrupan las especies de resinosas que tienen propiedadas mecánicas similares.

Estos grupos son:

\begin{tabular}{|c|c|}
\hline$\sigma_{\mathfrak{l}}$ & $\begin{array}{l}\text { Pino de Oregón (Pseudotsuga douglasii). } \\
\text { Alerce (Larix decidua). } \\
\text { Pino palustre (Pinus palustris\}. } \\
\text { Pino silyastre (Pinus sylvistols). }\end{array}$ \\
\hline $\mathrm{G}_{2}$ & 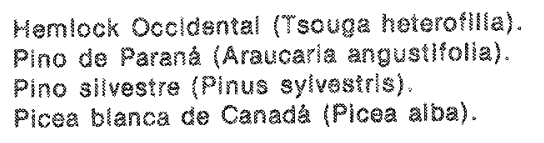 \\
\hline$\sigma_{3}$ & $\begin{array}{l}\text { Abeto rojo europeo (Picea abies). } \\
\text { Picea plateada de Canadá (Picea sitchensis. } \\
\text { Cedro rolo de Canadá Thuja plicata). }\end{array}$ \\
\hline
\end{tabular}

Las maderas de frondosas debidas a su gran variedad, no permiten el agruparas, debiendo partirse para su uthización de las caracteristicas mecánicas de cada una de ellas.

Una estructura laminada con madera de densidad me dia de $450 \mathrm{~kg} / \mathrm{m}^{3}$ (tundamentalmente maderas de resinosas) presenta características mecánicas bastante elevadas.

En las tabas siguientes se dan las tensiones aplican bles a las maderas de conferas de los grupos $G_{1}, G_{2} y$ $\mathrm{G}_{3}$ en estado seco (humedad de la madera $<18 \%$ y en estado verde.

En la segunda columa de las tablas siguientes se expresa, para cada grupo de mederas, las diew rentes clases de maderas según la Noma Españo. la de madera aserrada.

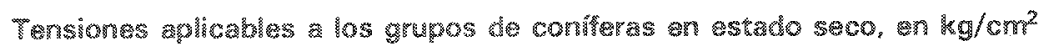

\begin{tabular}{|c|c|c|c|c|c|c|c|}
\hline \multirow{2}{*}{ Gropo } & \multirow{2}{*}{ Cabicatión } & \multirow{2}{*}{$\begin{array}{c}\text { Fiexions paralek } \\
\text { la pibra }\end{array}$} & \multirow{2}{*}{$\begin{array}{c}\text { Comprastom pardicia } \\
\text { a la thbra }\end{array}$} & \multirow{2}{*}{$\begin{array}{c}\text { Compresion } \\
\text { gargendicutar at a } \\
\text { figra }\end{array}$} & \multirow{2}{*}{ 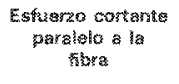 } & \multicolumn{2}{|c|}{ Motulute te elasticidads } \\
\hline & & & & & & Medio & Miramo \\
\hline \multirow{5}{*}{$G_{\xi}$} & Básica & 176 & 134 & 25 & 15 & \multirow{5}{*}{98.500} & \multirow{5}{*}{49.200} \\
\hline & 0.75 & 132 & 95 & 22 & 12 & & \\
\hline & 0,85 & 11 旗 & 77 & 22 & 10 & & \\
\hline & 0,50 & 88 & 45 & 20 & 8 & & \\
\hline & 0,40 & 70 & 45 & 20 & 6 & & \\
\hline \multirow{6}{*}{$\sigma_{2}$} & Básica & 140 & 112 & 21 & 15 & \multirow{6}{*}{84.400} & \multirow{6}{*}{49.200} \\
\hline & 0,75 & 98 & 81 & 18 & 12 & & \\
\hline & 0,65 & 81 & 87 & 18 & 10 & & \\
\hline & 0,50 & 63 & 49 & 15 & 8 & & \\
\hline & 0,40 & 53 & 39 & 15 & 6 & & \\
\hline & Básica & 105 & 84 & 15 & 13 & & \\
\hline \multirow{4}{*}{$\mathrm{G}_{3}$} & 0.75 & 67 & 53 & 13 & 9 & \multirow{4}{*}{70.300} & \multirow{4}{*}{38.700} \\
\hline & 0,65 & 56 & 42 & 13 & 8 & & \\
\hline & 0,50 & 45 & 32 & $\uparrow 1$ & 6 & & \\
\hline & 0.40 & 35 & 25 & 11 & 5 & & \\
\hline
\end{tabular}




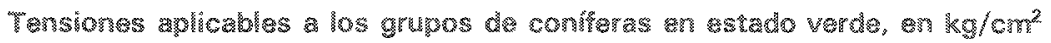

\begin{tabular}{|c|c|c|c|c|c|c|c|}
\hline \multirow{2}{*}{ Gruso } & \multirow{2}{*}{ Cellitisacion } & \multirow{2}{*}{ 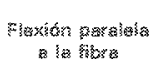 } & \multirow{2}{*}{ 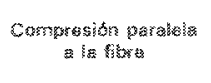 } & \multirow{2}{*}{ 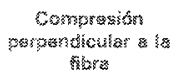 } & \multirow{2}{*}{ 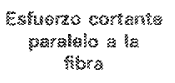 } & \multicolumn{2}{|c|}{ Rrobuto do alasticidad } \\
\hline & & & & & & Medis & Mistrue \\
\hline \multirow{5}{*}{$G_{1}$} & Básice & 140 & 98 & 18 & 14 & \multirow{5}{*}{91.400} & \multirow{5}{*}{45.700} \\
\hline & 0,75 & 105 & 74 & 15 & 11 & & \\
\hline & 0,65 & 9 & 64 & 5 & 9 & & \\
\hline & 0,50 & 70 & 49 & 13 & 7 & & \\
\hline & 0,40 & 56 & 39 & 13 & 6 & & \\
\hline \multirow{6}{*}{$\sigma_{2}$} & Básica & 112 & 84 & 16 & Tै। & \multirow{6}{*}{70.300} & \multirow{6}{*}{42.200} \\
\hline & 0,75 & 84 & 63 & 12 & 11 & & \\
\hline & 0,65 & 73 & 55 & 12 & 9 & & \\
\hline & 0,50 & 56 & 42 & 11 & 7 & & \\
\hline & 0,40 & 45 & 34 & 13 & 6 & & \\
\hline & Básica & 77 & 56 & 11 & 11 & & \\
\hline \multirow{4}{*}{$G_{3}$} & 0.75 & 58 & 42 & 9 & 8 & \multirow{4}{*}{59.800} & \multirow{4}{*}{31.600} \\
\hline & 0,05 & 50 & 37 & 9 & 8 & & \\
\hline & 0,50 & 39 & 28 & 8 & 6 & & \\
\hline & 0,40 & $3 !$ & 22 & 8 & 4 & & \\
\hline
\end{tabular}

Procadmententws generales de cấleulo

La determinación de las fuerzas de sección y de las tensiones que originan se realiza aplicando los méto. dos clásicos de cálculo, desarrollados en los tratados de Resistencia de Materiales.

Las tensiones admisibles que se aplican en los cálou. los vendrán afectadas por una serie de coeficientes que pasamos a enumerar:

1. Máximo coeticiente reductor por defecto de las láw minas (ver vablas siguientes).
Observamos en esas tablas una clara ventala que pre senta la madera laminada frente a la mader maciza. y as que, por el hecho de la laminación, la madera mejo. ra sensiblemente sus propiedades mecánicas al producirse una dispersión de los defectos, con lo que su inmuencia an las propledades de la pieza son mucho menores. Asi, vemos que una madera cuya calidades 0,75 (sus tensiones admisibles son el $75 \%$ de las bá sicas) por al hecho de laminarse pasa a ser de calidar 1. De forma análoga sucede cuando procedemos a la combinación de maderas de distintas calidades, prom duciendose también una dispersión de los defectos y aumentando la calidad mecánica de la pieza.

a) Utilizando láminas de madera de la misma calidad.

\begin{tabular}{|c|c|c|c|c|c|c|}
\hline 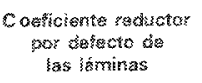 & No do lám: & 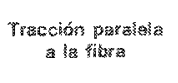 & $\begin{array}{l}\text { Mótuldo de slasticidad } \\
\text { on fiention }\end{array}$ & 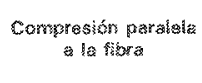 & 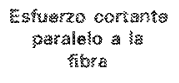 & 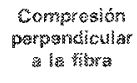 \\
\hline 0,75 & 46 más & 1,00 & 1,00 & 1,00 & 0.90 & 1,00 \\
\hline \multirow{8}{*}{0,65} & $s$ & 0,68 & 0.88 & 0,87 & \multirow{8}{*}{0,90} & \multirow{8}{*}{1,00} \\
\hline & 5 & 0,72 & 0,89 & 0,89 & & \\
\hline & 10 & 0,73 & 0.91 & 0,91 & & \\
\hline & 15 & 0,80 & 0,82 & 0,92 & & \\
\hline & 20 & 0,82 & 0,93 & 0,93 & & \\
\hline & 30 & 0,84 & 0,94 & 0,93 & & \\
\hline & 50 & 0.85 & 0,94 & 0,94 & & \\
\hline & 100 & 0.88 & 0,95 & 0,95 & & \\
\hline \multirow{8}{*}{0,50} & s & 0,40 & 0.77 & 0,76 & \multirow{8}{*}{0,90} & \multirow{8}{*}{1,00} \\
\hline & 5 & 0,44 & 0,72 & 0.78 & & \\
\hline & 10 & 0,53 & 0,82 & 0,82 & & \\
\hline & $\$ 5$ & 0,58 & 0.83 & 0.83 & & \\
\hline & 20 & 0,60 & 0,8 & 0,34 & & \\
\hline & 80 & 0,68 & 0,86 & 0,85 & & \\
\hline & 50 & 0.68 & 0,87 & 0.87 & & \\
\hline & 100 & 0,70 & 0,88 & 0,88 & & \\
\hline
\end{tabular}




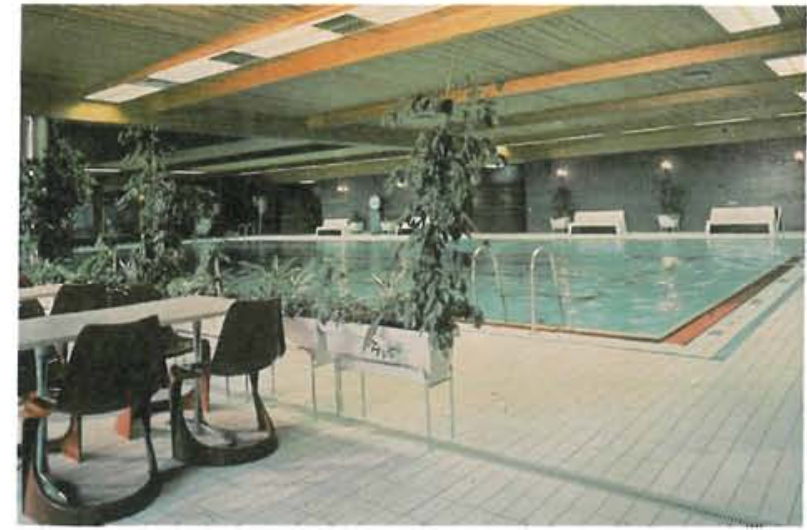

Complejo deportivo Dureholms halle en Katrineholm (Suecia).
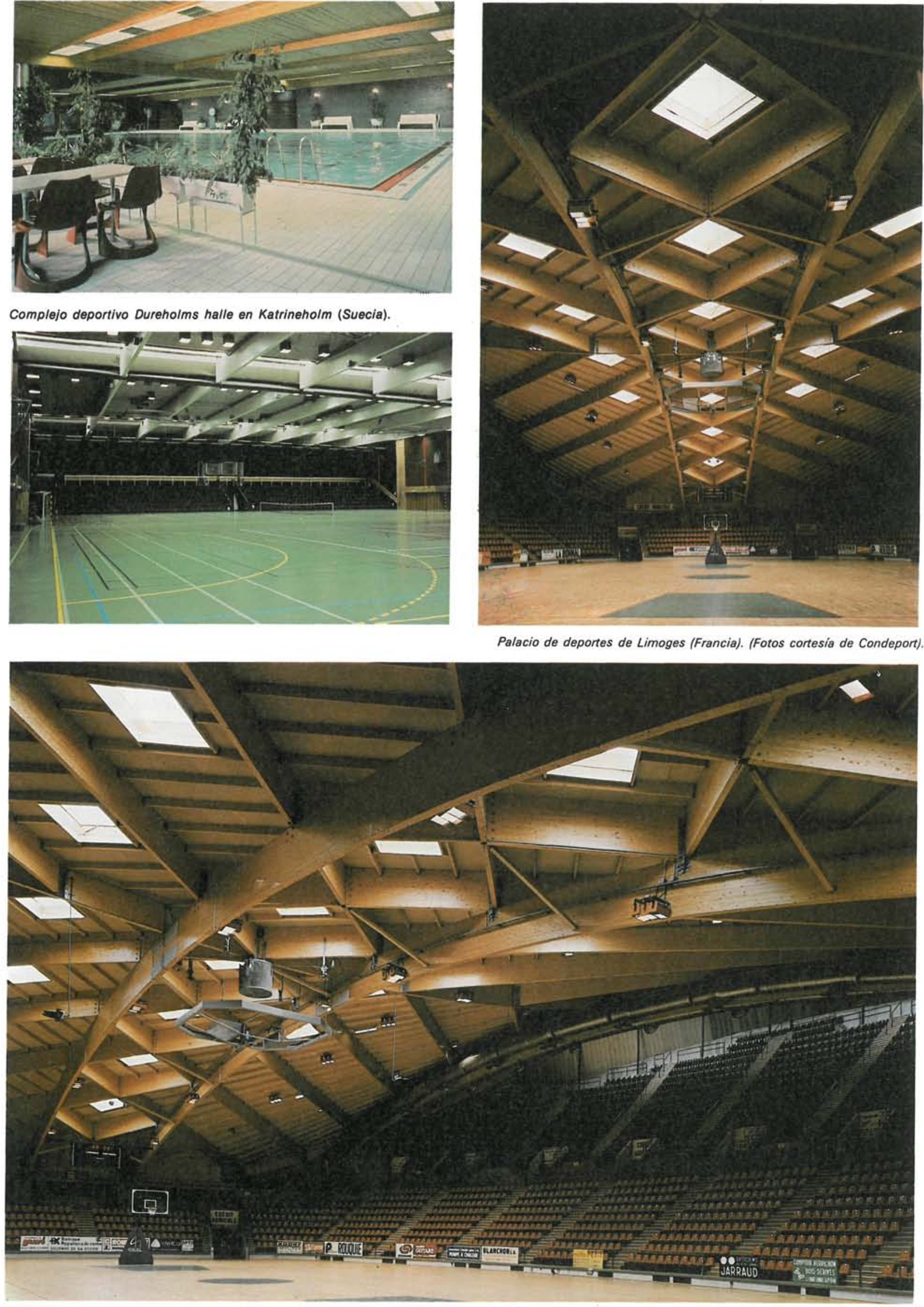

Palacio de deportes de Limoges (Francia). (Fotos cortesía de Condeport). 


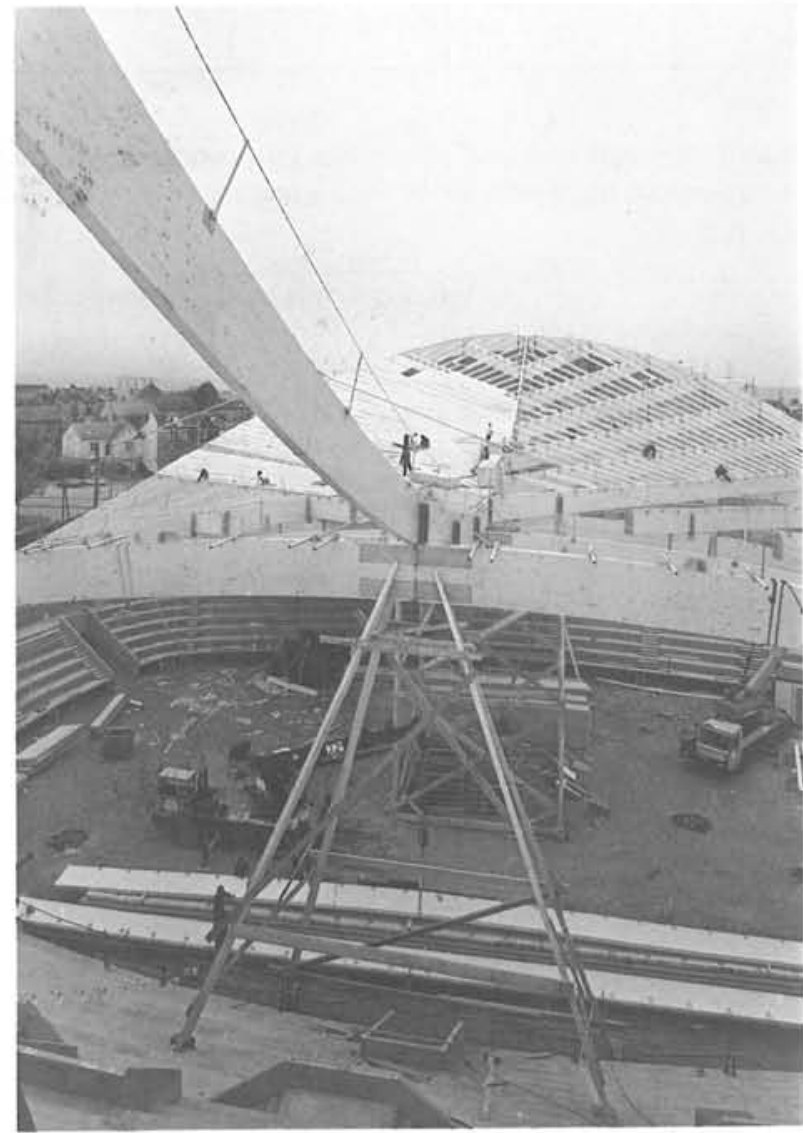

Palacio de deportes de Limoges (Francia). (Fotos cortesia de CONDEPORT.

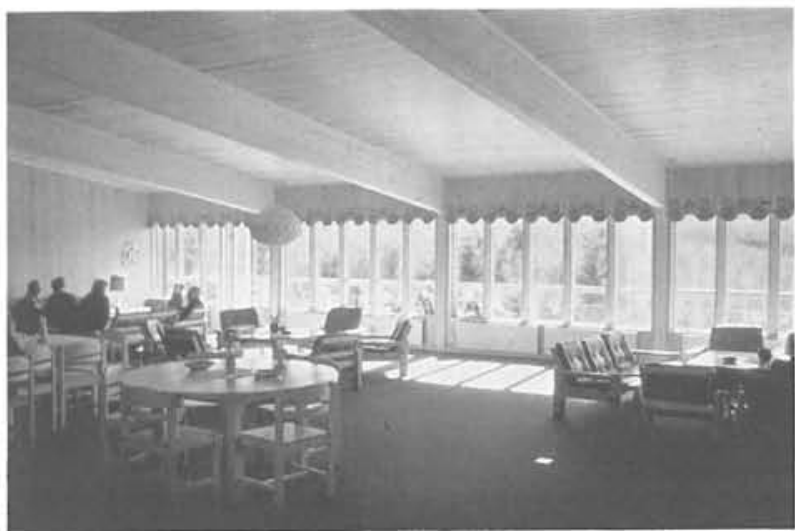

Sala de conferencias. (Foto cortesía de TOREBODA LIMTRA).

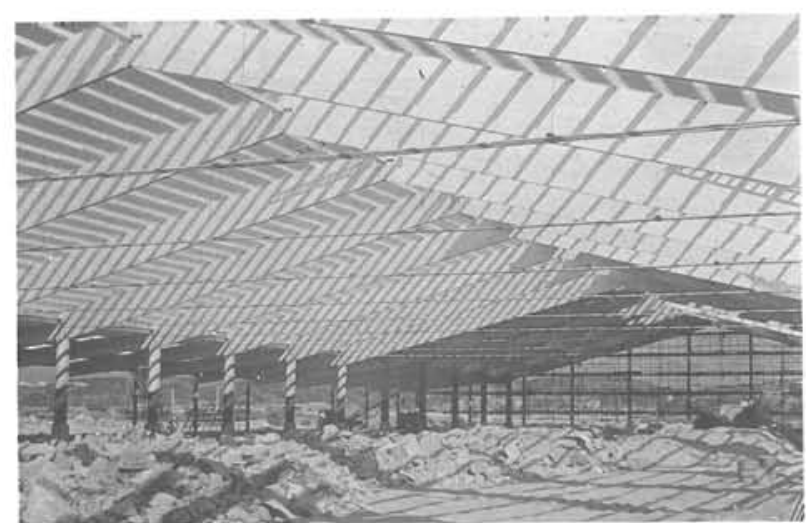

Estación terminal en Gothemburg. (Foto cortesía de TOREBODA LIMTRA).
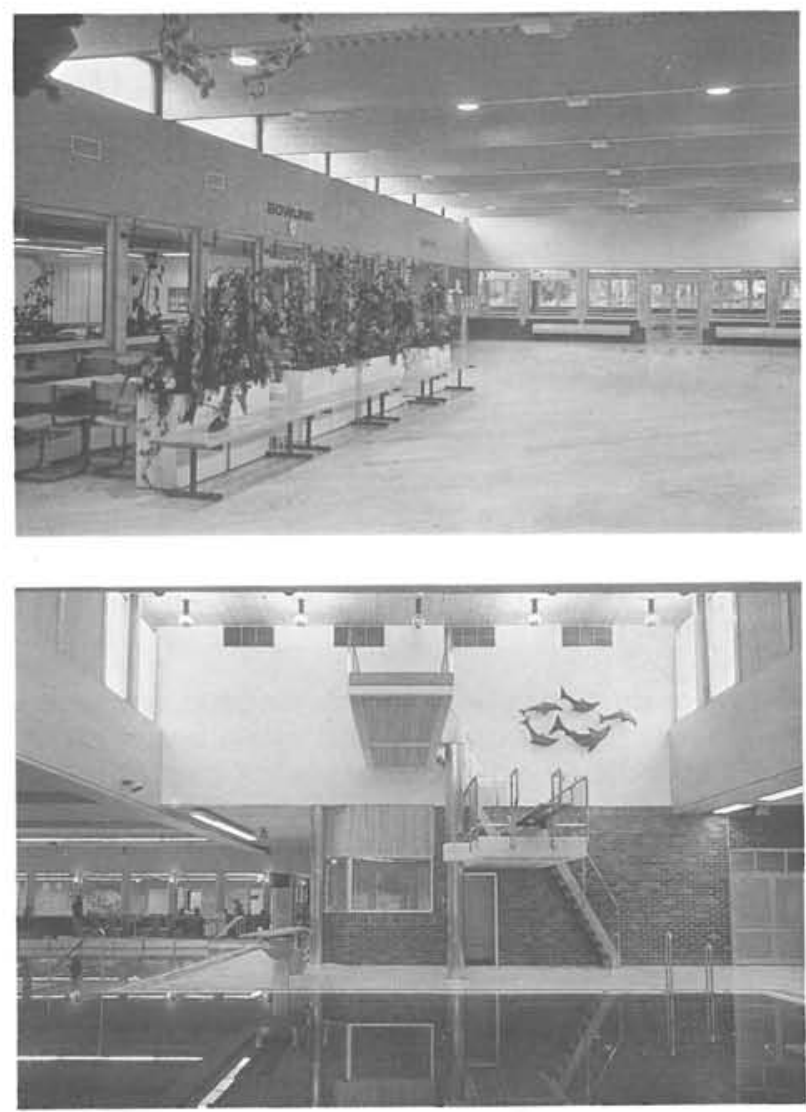

Fotografias del complejo deportivo cubierto "Duveholmshalle" en Katrineholm (Suecia). (Cortesia de KURT EVAN SONEHAG). 
b) Utilizando combinaciones de laminas de distinta caldad.

\begin{tabular}{|c|c|c|c|c|c|c|}
\hline Composisción & No de lántromas & $\begin{array}{c}\text { Tracción pazalela } \\
\text { a f a sibra }\end{array}$ & 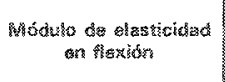 & 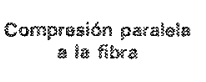 & 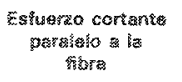 & 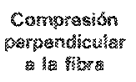 \\
\hline 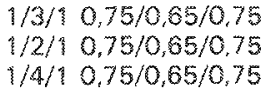 & 48 más & 0,95 & 0,98 & 0,93 & 0.90 & 1.00 \\
\hline $\begin{array}{l}1 / 2 / 10,65 / 0,50 / 0,65 \\
1 / 3 / 1 \\
1 / 4 / 1\end{array}$ & $\begin{array}{c}4.5 \times 6 \\
10 \\
15 \\
20 \\
30 \\
50 \\
100\end{array}$ & $\begin{array}{l}0,68 \\
0,74 \\
0,78 \\
0.78 \\
0,80 \\
0,82 \\
0,84\end{array}$ & $\begin{array}{l}0,88 \\
0,90 \\
0,91 \\
0,92 \\
0,92 \\
0,93 \\
0,94\end{array}$ & $\begin{array}{l}0,82 \\
0,85 \\
0,86 \\
0,87 \\
0,88 \\
0,89 \\
0,90\end{array}$ & 0,50 & 1.00 \\
\hline
\end{tabular}

2. Coeficiante según la duración de la carga para piezas sometidas a flexión.

Las tensiones también vienen modificadas según la duración de las cargas, pudiendo soportar las estructuras cargas mayores a las admisibles para larga duración cuando éstas actúen un tiempo relativamente cotto.

\begin{tabular}{|c|c|}
\hline Duración da la carge & Coet3cians: \\
\hline $\begin{array}{l}\text { De larga duración lpeso propio y cargas pemaw } \\
\text { nentes! } \ldots \ldots \ldots \ldots \ldots \ldots \ldots \ldots \ldots \ldots \ldots\end{array}$ & 1.00 \\
\hline $\begin{array}{l}\text { De ouracion media lpeso propio }+ \text { nieve, peso } \\
\text { propio } \neq \text { cargas eventuales } \ldots \ldots \ldots \ldots \ldots\end{array}$ & 1.25 \\
\hline $\begin{array}{l}\text { De cona duración lpeso propio + cargas de mpac } \\
\text { to to vientol } \ldots \ldots \ldots \ldots \ldots \ldots \ldots \ldots \ldots\end{array}$ & 1,50 \\
\hline
\end{tabular}

3. Coeficiente segun la longitud y posición de los apoyos.

En cualquier apoyo de carga perpendicular a la tibra. las tensiones admisibies en compresión perpendicular a la fibra dependen de la longitud y localización del arovo.

Las tensiones básicas son admisibles en compresión perpendicular a la fibra, para los soportes de cualquier longitud en los extremos de las plezas y para soportes de 15 o más centimetros de longitud, en cualquier po. sición de la pieza.

Er apoyos de menos we $15 \mathrm{~cm}$ de longitud, situados a 8 cm o más de los extremos de la pieza, las tensiones producidas deben multiplicarse por los coeficientes:

\begin{tabular}{|l|c|c|c|c|c|c|c|}
\hline Longitud del apoyo $(\mathrm{cm})$ & 1,5 & 2,5 & 4,0 & 5,0 & 8,0 & 10 & 15 \\
\hline Cosíciente & 1,70 & 1,53 & 1,36 & 1,19 & 1,14 & 1,10 & 1,00 \\
\hline
\end{tabular}

4. Cargas formando ángulo con la fibra de la ma. dera:

Cuando la dirección del estuezo forma un cierto ángu. lo con las fibras de madera en el elemento que se con- sidere, carga unitara admisible en compresión para la superficie incinada es la siguiente:

$$
C_{C \theta}=\frac{C_{C I I} \cdot C_{c 1}}{C_{C I I} \cdot \operatorname{sen}^{2} \theta+C_{C L} \cdot \cos ^{2} \theta}
$$

Siendo:

$\mathrm{C}_{\mathrm{Cl}}=$ = carga admisible paralela a la tibra.

$C_{\mathrm{cl}}=$ carga admisible perpendicular a la sibra.

$\mathrm{C}_{0}$ : $=$ carga amisible an la superticie inelmeda.

$y=$ ángulo entre la dirección del esfuerzo y la direc ción de las fibras.

5. Estueros cortantes en elementos estructurales tra bajando a flexion, rebajados en los extremos.

El esfuerzo cortante en los extremos rebajados en los clementos sometidos a Hexión, será el correspondien. te a la tensión calculada por su clasificación multiplicada por el coeficiente:

$$
c=\frac{\text { Alura gtectiva }}{\text { Altura total }}
$$

6. Coeficiente de forma para elementos sometidos a flexión.

Las tensiones de clasificación se aplican a elementos de madera sólida o de madera laminada, cuya sección sea rectangular.

Para piezas laminadas de alura superior a $80 \mathrm{~cm}$ las tensiones vienen multiplicadas por ef factor de forma:

$$
c=\frac{d+922,6}{d+568}, d \text { expresado en } \mathrm{cm}
$$

Para atras fomes se han de aplicar los correspondientes factores correctores, asi:

- Circular. ................... Coef. 1,18

- Sección cuadrada con cargas según

las diagonales.............. Coef: 1,41

7. Elemertos curvos.

Para las partes curvas de une pieza laminada, ta tensión de clasificación debe muttiolicarse por un coefi. 
ciente que exprese la relación entre el espesor y el ra. dio de curvatura: C/R.

\begin{tabular}{|l|l|l|l|l|l|l|l|l|}
\hline Relación C/R & $1 / 100$ & $1 / 125$ & $1 / 150$ & $1 / 175$ & $1 / 200$ & $1 / 250$ & $1 / 300$ \\
\hline Coeficiente & 0,80 & 0,87 & 0,80 & 0,91 & 0,93 & 0,84 & 0,95 \\
\hline
\end{tabular}

\section{Esbelrez}

Las tensiones de clasificación en elementos somein." dos a compresión deben modificarse en función de las condiciones de duración de las cargass y la esbeltaz de las piezas, con arreglo a los siguientes coelicientes:

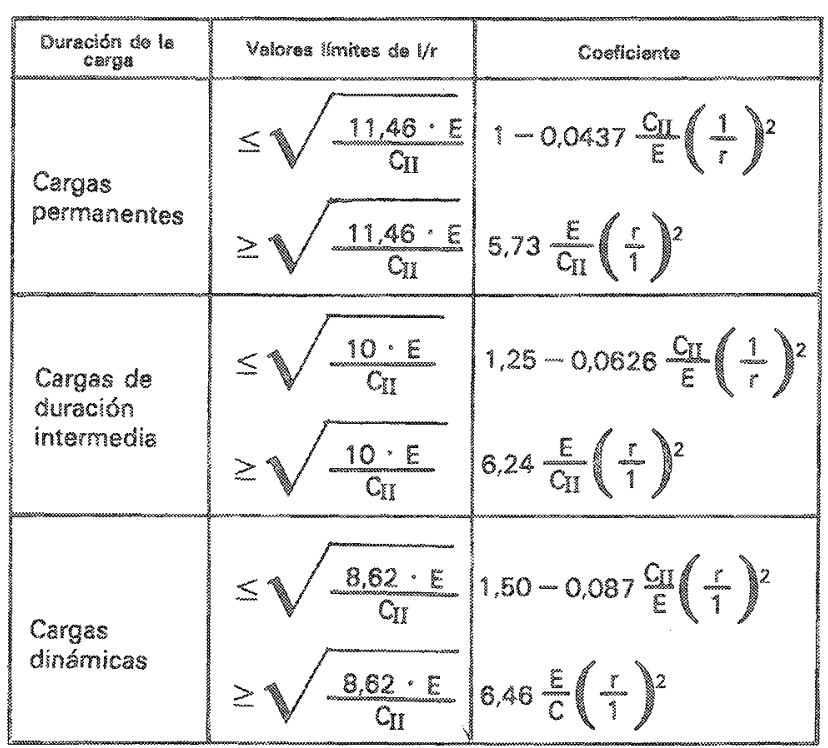

siendo:

$$
\begin{aligned}
& r=\text { dimensión transversal menor } \\
& 1=\text { longitud de la pieza } \\
& E=\text { módulo de clasticidad } \\
& C=\text { tonsión de compresión paralela a la fora }
\end{aligned}
$$

\begin{tabular}{|c|c|c|c|c|}
\hline \multicolumn{2}{|l|}{ Extsets 42} & \multicolumn{3}{|c|}{ Volores the cotritherta } \\
\hline$\| /$ & $1 / B$ & $\begin{array}{c}\text { Carmes } \\
\text { parmanoryte }\end{array}$ & $\begin{array}{c}\text { Cergas do } \\
\text { duracion moda }\end{array}$ & 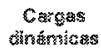 \\
\hline $\begin{array}{r}\text { Wenos de } \\
\\
10 \\
5\end{array}$ & $\begin{array}{l}1.40 \\
1.40 \\
2.90\end{array}$ & $\begin{array}{l}1,00 \\
0.99 \\
0.98\end{array}$ & $\begin{array}{l}1,25 \\
1.24 \\
1.23\end{array}$ & $\begin{array}{l}1.50 \\
1.49 \\
1.47\end{array}$ \\
\hline $\begin{array}{l}20 \\
30 \\
40\end{array}$ & $\begin{array}{r}5.80 \\
8,70 \\
11.50\end{array}$ & $\begin{array}{l}0,98 \\
0,94 \\
0,81\end{array}$ & $\begin{array}{l}1.20 \\
5.17 \\
5.13\end{array}$ & $\begin{array}{l}1,44 \\
1,40 \\
1,34\end{array}$ \\
\hline $\begin{array}{l}30 \\
60 \\
70\end{array}$ & $\begin{array}{l}14,40 \\
17,30 \\
20,20\end{array}$ & $\begin{array}{l}0.87 \\
0.83 \\
0.7 \%\end{array}$ & $\begin{array}{l}1,08 \\
1,00 \\
0,90\end{array}$ & $\begin{array}{l}1,27 \\
1,10 \\
1,01\end{array}$ \\
\hline $\begin{array}{r}90 \\
90 \\
100\end{array}$ & $\begin{array}{l}23.00 \\
28.00 \\
28.80\end{array}$ & $\begin{array}{l}0,70 \\
0,61 \\
0,53\end{array}$ & $\begin{array}{l}0.79 \\
0.68 \\
0.58\end{array}$ & $\begin{array}{l}0,86 \\
0,72 \\
0,60\end{array}$ \\
\hline $\begin{array}{l}120 \\
140 \\
160\end{array}$ & $\begin{array}{l}84,60 \\
40,40 \\
46,20\end{array}$ & $\begin{array}{l}0,40 \\
0,31 \\
0.24\end{array}$ & $\begin{array}{l}0,42 \\
0,32 \\
0,25\end{array}$ & $\begin{array}{l}0,44 \\
0,33 \\
0,25\end{array}$ \\
\hline $\begin{array}{l}180 \\
200 \\
200 \\
240 \\
250\end{array}$ & $\begin{array}{l}52,00 \\
57,70 \\
63,50 \\
69,20 \\
72,20\end{array}$ & $\begin{array}{l}0,20 \\
0,16 \\
0,13 \\
0,11 \\
0,10\end{array}$ & $\begin{array}{l}0,20 \\
0,16 \\
0,12 \\
0,12 \\
0,11\end{array}$ & $\begin{array}{l}0.20 \\
0,17 \\
0,14 \\
0,12 \\
0,11\end{array}$ \\
\hline
\end{tabular}

9. Coeficiente de esbeitez para maderas clasificadas 0,50 Y 0,80 .
Para el cálculo de las tensiones admisibles no se aplicarán de forma acumulativa todos los coeficientes, sino que se avaluarán los que htevienen, según el tipo de elemento estructural de que se trate, y se aplicará el menor da todos ollos, salvo los coeficientes de forma y esbeltez que se aplican siempre.

\section{Elecron de las fornas}

Por su fabricación, la sección transversal de los elementos es generaimente rectangular, buscándose una relación $h / b$ elevada a fin de obtener una inercia máxima con una sección dada.

Las anchuras habiuamente utilizadas dependen de las dimensiones comerciales de la madera aserrada. Las más frecuentemente empleatas son $11,5,13,5 \mathrm{y}$ $\$ 6 \mathrm{~cm}$.

La elección de la forma y de la sección es uno de los elementos importantes en el estudio económico de un proyecto.

Los elementos estructurales actualmente más emplea. dos en madera leminada son los siguientes (ver cuadro en la página siguiente).

\section{Vigas rectas}

Bajo el punto de vista económico, la usilización de vigas rectas supone un cierto ahorro de madera y cola en la fabricación de los elementos, sin embargo esta solución limita las luces a una troinena de matros.

En lo que concieme a su utilización, una solución inte. resante es el empleo de elementos standard pretabri. cados de débil sección (por ejemplo, hasta $50 \mathrm{~cm}$ de altural a fin de reemplazar la madera maciza por la la minada, así cono también los materides tradicionales de construcción, pues la ligereza es mucho mayor.

\section{Pơricos y arces}

Aparte de las vigas laminadas, son los pónticos y arcos los tipos estructurales más empleados en las construc: ciones de madera laminada.

En general, tanto pórticos como arcos, se suelen hacer triarticulados -..con rótulos en arranque y clave-- para facilitar su montaje.

Los pórticos a dos aguas triarticulados se hacen de sección variable, con espesor máximo en la iniciación del dintel y coronaclon del pllar, y minimo en aranques y clave. Naturalmente, todas las secciones inter medias entre arranques y claves están sometidas a compresión excéntrica, y sus secciones transuersales deben ser capaces de resistir estas solicitaciones. En arranques y clave, es el esfuerzo cortante el que predo. mina. En la práctica, el canto en aranque y clave viene a estar comprendido entre $5 / 8$ y $3 / 4$ del espesor co. 


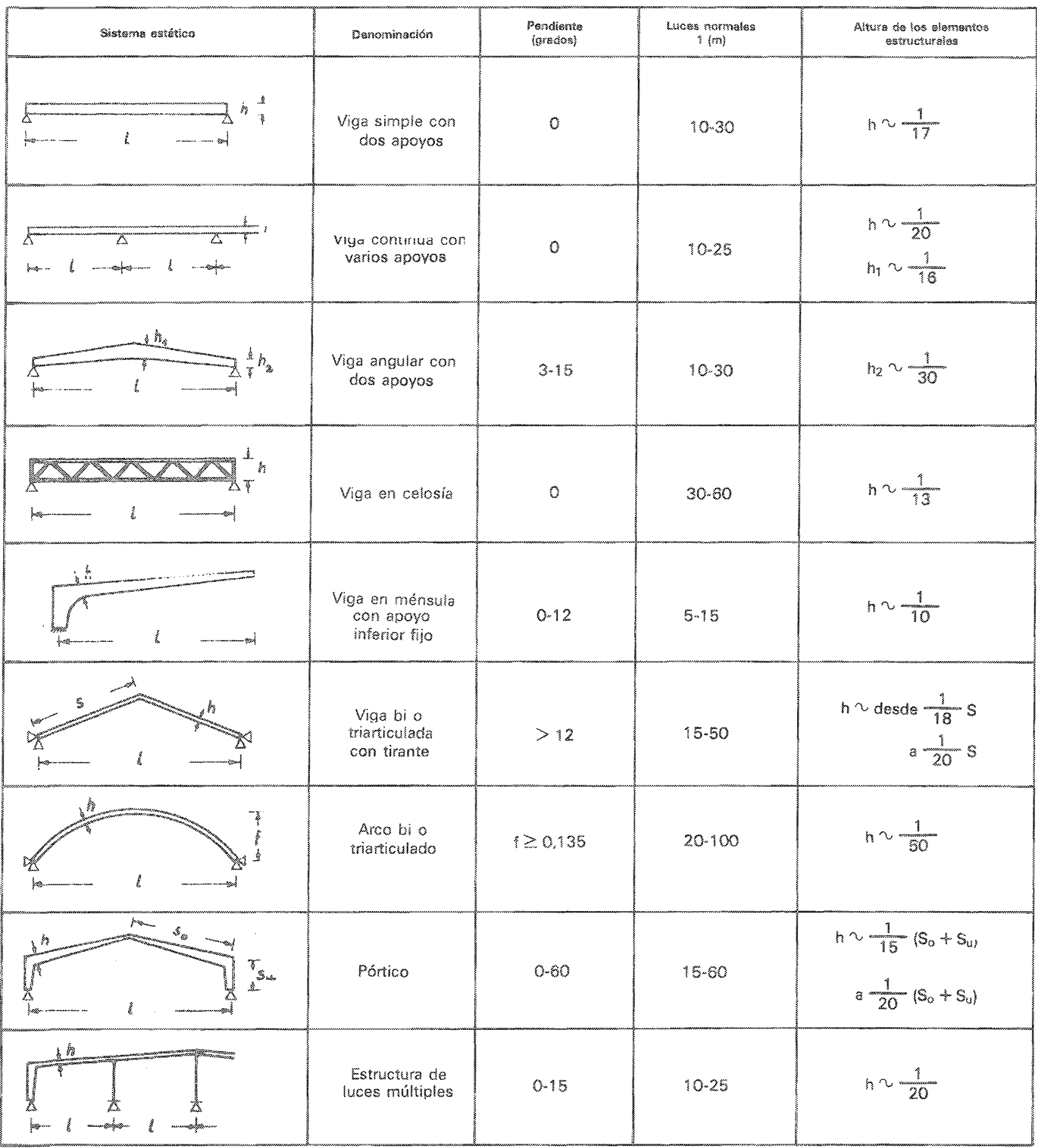

Siendo de éstos los más ampliamente empleados las vigas rectas, los arcos y póticos.

rrespondiente a las secciones extremas de la zona cur vada. Estas dimensiones pueden ser más reducidas, pero no es comeniente, ya que a veces se alcanzan dotormaclones impontantes, y ademés, hay oue verficar gran numero de secciones intermedias.

Cuando se proyeckan arcos, éstos suelen ser de sección constante en roda longitud. Si el faldón tiene po. ca inclinacion, el radio de curature del codo es mu pequeño: y en este caso la solución economica para conseguir radios de curvatura mayores consiste en sostener pare de la cubienta mediante una viga incli" nada que se apoya en el dintel y soporte.

\section{Urionsus}

Las uniones entre los diversos elementos constitutivos de una estructura henen funciones que deben ser ar. monizadas con las hunciones corraspondientes de los dementos laminados.

Une de las funciones fundamentales es de carácter mecánico $y$ consigte en transmitir los esfueros, bien 
de una pieza a otra, o bien de una pieza a la infraestructura de los soportes.

El ensamblaje entre las piezas de unión y la madera se realiza por bulones perpendiculares al eje de la pieza y paralelos a sus planos de encolado. De una manera general, cada vez que se busca una gran rigidez en la unión, se utiliza mayor número de bulones de diámetro inferior. Esta rigidez puede ser necesaria, por ejemplo, para limitar los deslizamientos en el caso de estructuras donde el cúmulo de deslizamientos en un gran número de ensamblajes podría perjudicar la estabilidad de la estructura, o incluso en el caso de arcos de muy débil flecha en los que un ligero deslizamiento puede afectar profundamente el tipo de funcionamiento de la estructura (paso de funcionamiento como arco comprimido, a un funcionamiento de viga en flexión). Cuando la pieza de unión asegura una articulación, ésta se realiza generalmente en dos piezas ensambladas por un eje, cuyo dimensionamiento se hace en función de las reglas clásicas de construcción.

Una excelente precaución consiste en no asegurar la transmisión de los esfuerzos más que por intermedio de bulones $y$ órganos complementarios sin tener en cuenta contactos directos entre el herraje y la madera.

Hay que señalar igualmente que las reglas actuales precisan la necesidad de una simetría de los ensamblajes con relación al plano medio de cada pieza ensamblada.

En las figuras siguientes se detallan las uniones más utilizadas en los distintos tipos de estructuras.

En la figura 1 se representa la forma de realizar una rótula perfecta, utilizando piezas construidas con chapas de acero y unidas por un pasador.
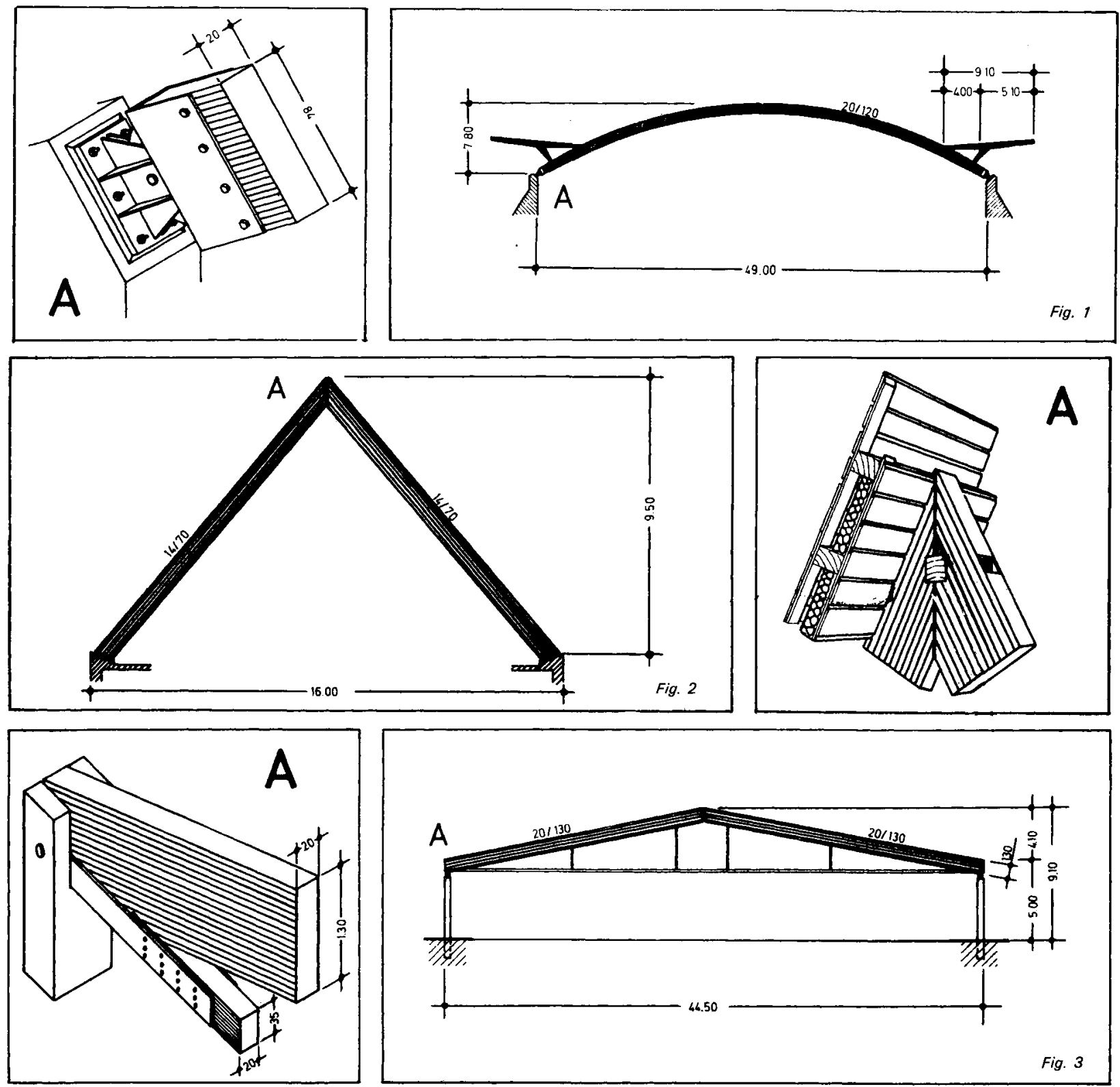
La figura 2 corresponde a un pórtico con los faldones muy apuntados. Este dispositivo permite transmitir cargas horizontales y verticales.

La unión se hace empleando una pareja de llaves de disco, colocadas espalda con espalda, y un perno cuyas cabezas y arandelas se alojan en cajetines realizados en las piezas de madera. A veces los extremos de las piezas llevan un ligero chaflán para evitar su aplastamiento.

En los arcos y pórticos atirantados es muy importante el enlace del cordón con el tirante. La solución que muestra la figura 3 consta de una pieza metálica con la forma adecuada para recibir el empuje y transmitir al tirante el esfuerzo horizontal.

La unión de las correas con el dintel puede efectuarse, si las viguetas se colocan con su paramento superior enrasado con el dintel del pórtico o arco, mediante unas piezas especiales de acero, que sirven para alojar directamente la correa.

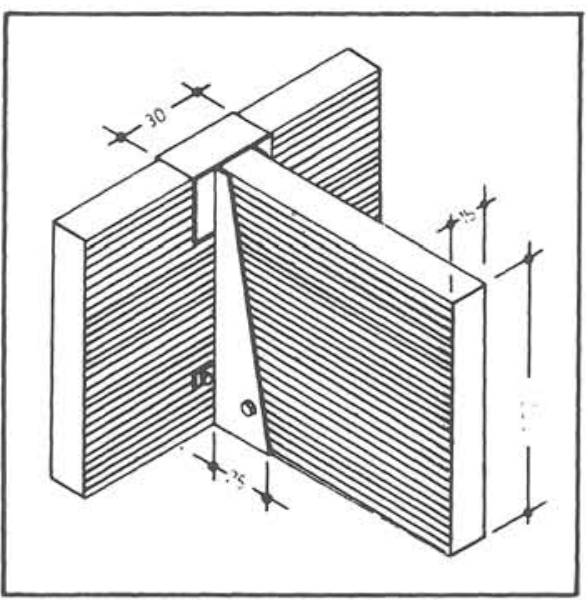

Fig. 4

Existe además otro tipo de uniones, destinadas a asegurar una continuidad en el material mismo, encontrándose dos variedades principales:

- La junta de transporte necesitada por las limitaciones impuestas al efectuar el transporte de los elementos por carretera.

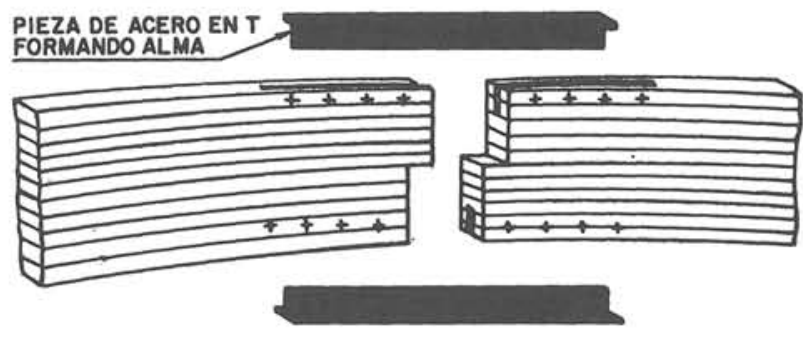

Fig. 5. - Junta de transporte para arco de media y gran luz.

- El ensamblaje en ángulo destinado a encastrar mutuamente dos elementos, en general rectos pero alineados, y que surge de la imposibilidad tecnológica de reducir a voluntad los radios de curvatura.

En el primer caso la unión se colocará, en la medida que sea posible, en un punto de momento nulo bajo el efecto de las cargas verticales. Sin embargo, la existencia de momentos de flexión bajo la acción del viento, conduce a prever los ensamblajes susceptibles de

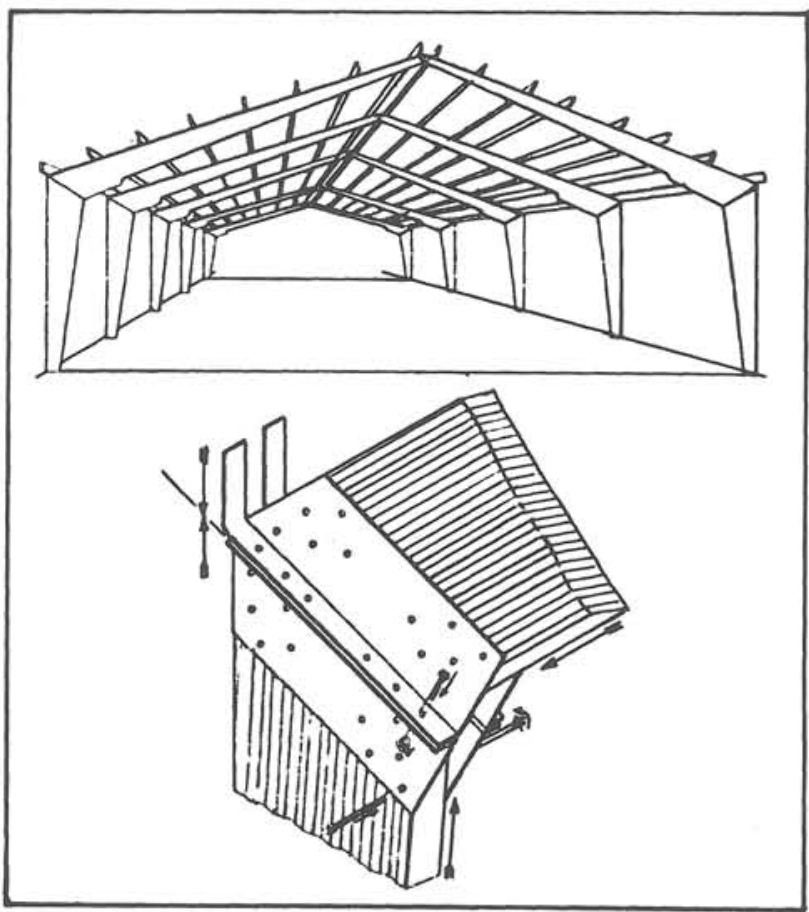

Fig. 7.-Detalle de ensamblaje en ángulo por uniones metálicas.
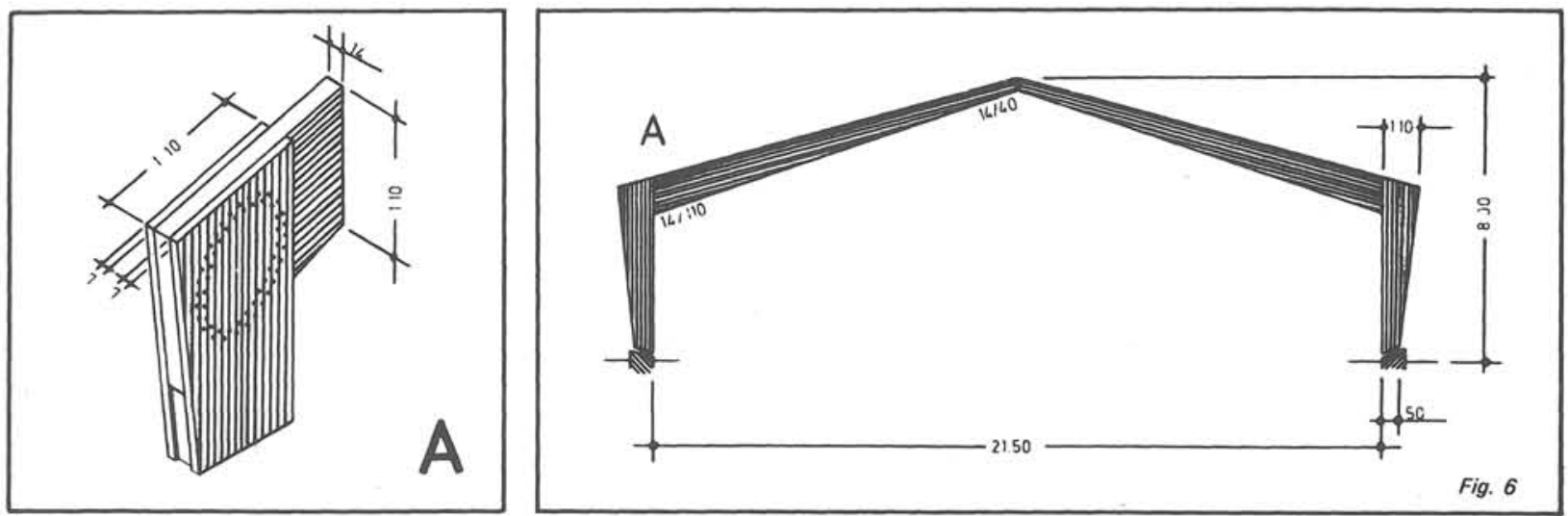
absorber tanto los esfuerzos de flexión como los normales.

En el segundo caso (uniones de ángulos) por el contrario, los momentos de flexión a absorber son los más intensos, y la concepción de la unión es uno de los puntos críticos de la construcción. En las figuras 6,7 , 8 y 9 se observan las soluciones constructivas más empleadas. Presentando la unión por entalladuras múl-

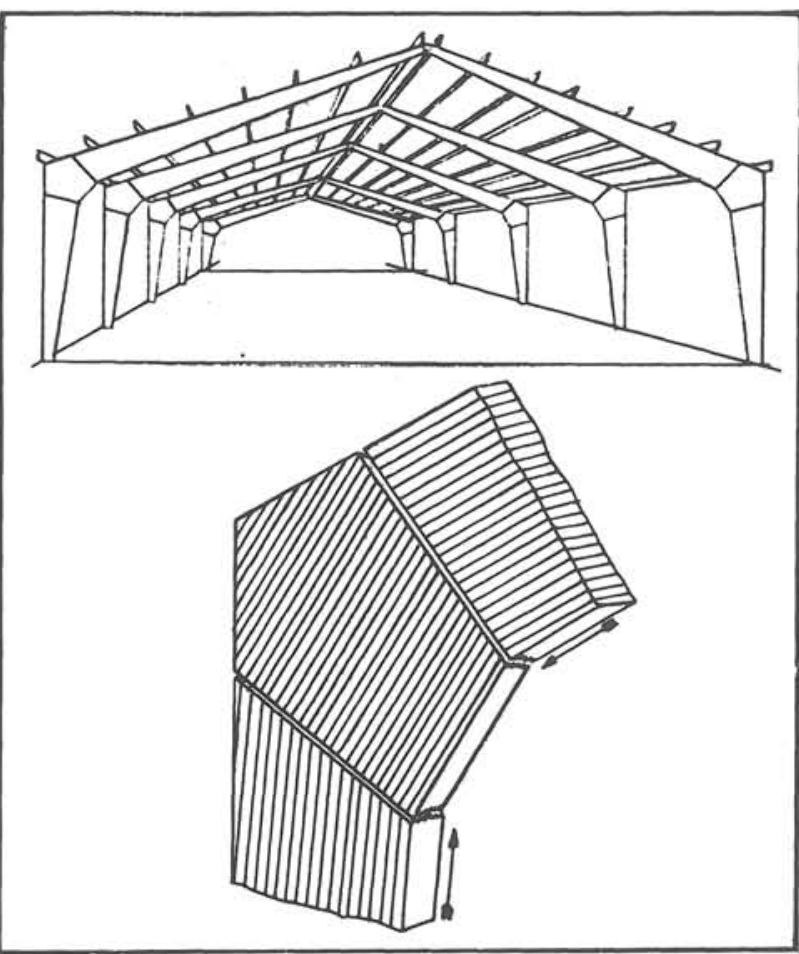

Fig. 8. -Detalle de ensamblaje en ángulo por cuña de madera laminada.

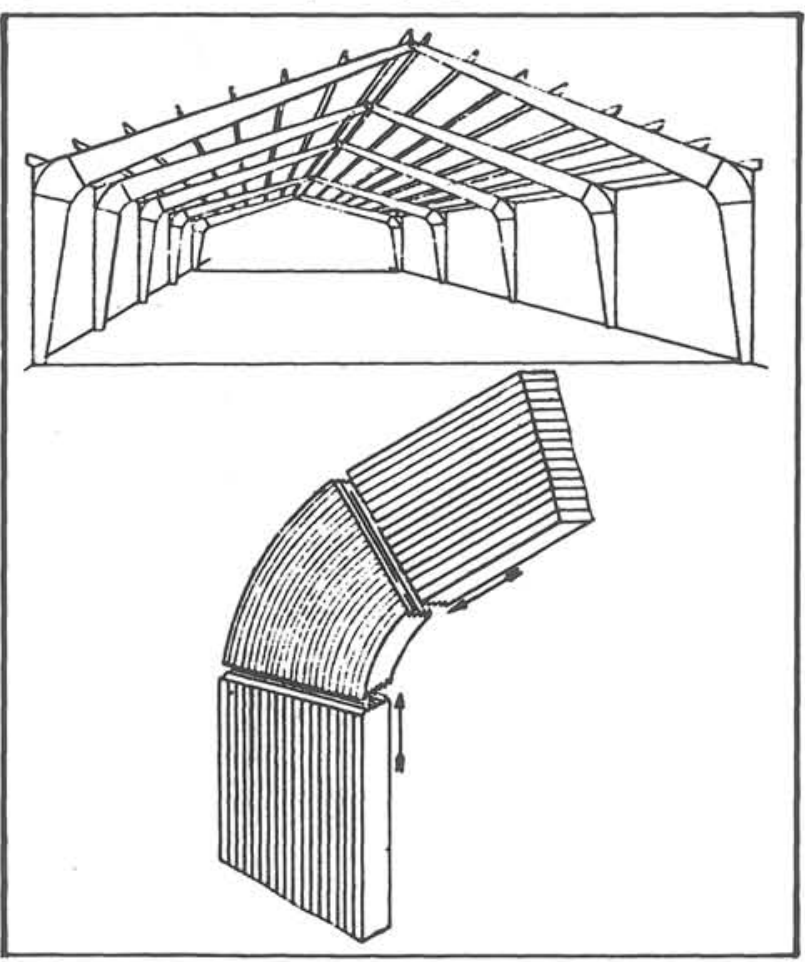

Fig. 9.-Ensamblaje por entalladuras múltiples. tiples, según la bisectriz del ángulo, en las juntas de las piezas encoladas hay una rigidez superior a la que se puede alcanzar mediante el empleo de elementos metálicos.

\section{Ventajas técnicas de las construcciones de madera laminada}

La madera laminada es, ante todo, madera y presenta por ello las cualidades bien conocidas propias de este material. Enumeraremos sucintamente las siguientes:

- Buenas características mecánicas debido a la baja densidad. Ello se traduce en cotas de calidad (relación de resistencia mecánica a la densidad) muy elevadas y del mismo orden que las de ciertas aleaciones ligeras.

- Un excelente comportamiento a la flexión y la compresión.

- Débil conductividad térmica.

- Ausencia de dilatación térmica.

- Variaciones dimensionales según el sentido axial (paralelo a las fibras) prácticamente despreciables bajo el efecto de las variaciones de humedad.

- Una gran estabilidad química.

- Características mecánicas muy poco variables en función de la temperatura.

- Una gran resistencia al fuego y un comportamiento perfectamente previsible.

- Gran facilidad de trabajo y ejecución de las uniones químicas y mecánicas (encolado, atornillado, clavazón, pernos, etc.) con la mayor parte de los materiales de construcción.

A estas propiedades hay que añadir las que se obtienen por la laminación, y que han sido enumeradas al comienzo de este artículo, deduciéndose del conjunto de estas características claras ventajas técnicas que no deben escapar a los diseñadores:

- La posibilidad de realizar grandes luces libres.

- La prefabricación, que si a veces es la causa de alguna preocupación para su transporte en los casos extremos, permite, por el contrario, racionalizar y reducir considerablemente los tiempos de montaje y puesta en obra. En efecto, al tratarse de elementos prefabricados relativamente ligeros, su puesta en obra raramente plantea problemas, obteniéndose un ahorro de tiempo muy apreciable si lo comparamos con el empleado en una construcción análoga con materiales tradicionales.

- La ligereza del conjunto de la estructura, siendo la relación del peso muerto a las cargas climáticas o de explotación más baja, a igualdad de luz que la de la mayor parte de los demás materiales, lo que conduce a un aligeramiento notable de las fundaciones, así como una economía global sobre la construcción y que permite, en ciertos casos, edificar una estructura sobre terrenos de consistencia más débil.

- La posibilidad de ampliaciones y modificaciones de edificios sin gran dificultad. 
- La excelente resistencia a los ataques químicos, lo que incide de forma creciente en la utilización de este tipo de estructuras para el almacenaje de productos activos y para la construcción de edificios que deben soportar una atmósfera salina. Esta importante propiedad hace a la madera laminada mucho más adecuada que el acero o el hormigón en edificios en los que los riesgos de corrosión son elevados, tales como: fábricas de productos químicos, papeleras, tintorerías, piscinas, construcciones agrícolas, silos de almacenaje de productos corrosivos, etc.

- La alta resistencia de la madera al fuego, la cual se encuentra incrementada por las secciones que generalmente se utilizan en las obras de madera laminada y que confiere a la estructura una resistencia que excede ampliamente las exigencias de las nor- mas de seguridad. Esta propiedad hace que sea cada vez más utilizada por los arquitectos cuando se requiere un alto nivel de seguridad, ya sea para seguridad de las personas como para permitir la evacuación eventual de equipos costosos; por otra parte, las compañías de seguros tienen la tendencia, cada vez más frecuente, a tener en cuenta en sus tarifas estas particularidades.

- Junto a estas ventajas hay que añadir las indiscutibles características estéticas de este tipo de material. Las estructuras de madera laminada tienen un aspecto generalmente muy agradable en razón de las curvaturas «naturales» que comportan, del carácter macizo de las secciones rectangulares utilizadas, de la variabilidad de las citadas secciones y del aspecto superficial. Factores estos que pueden ser ampliamente interpretados por los arquitectos.

publicaciones del I.E.T.C.C.

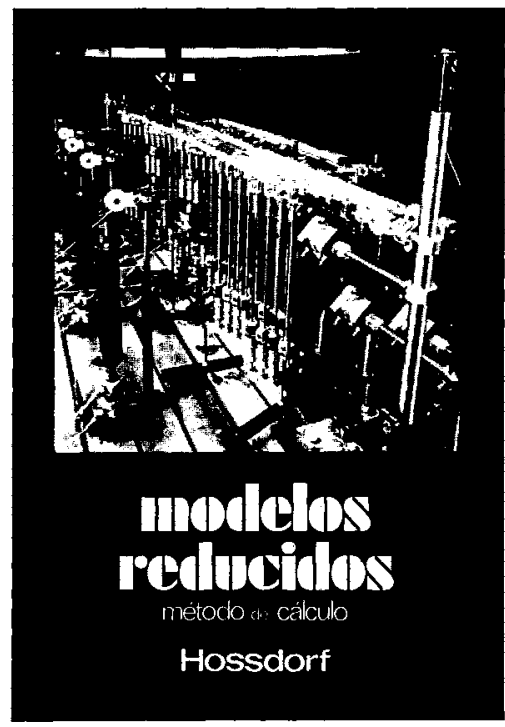

Modelos reducidos. Método de cálculo

H. Hossdorf, Ingeniero Civil

La técnica de los ensayos en modelos reducidos de estructuras sufre hoy dia una decisiva metamorfosis. Masta hace poco era un medio más bien de artesania, que no siempre era tomado en serio por los académicos teorizantes oara comprender el comporta miento resistente de las estructuras complejas y al que se acudió las más de las veces, como a un último remedio debido a sus indiscutibles insuficiencias. Sin embargo, en poco tiempo y gracias a su conexión con los ordenadores digitales, se ha transformado en un instrumento cientificamente valioso, que no puede quedar a un lado en la práctica diaria del Ingeniero Provectista.

Un volumen encuadernado en cartoné plastificado con tomo de tela, de $17 \times 24 \mathrm{~cm}$, compuesto de 250 páginas, 158 figuras y fotografias.

Precios: 1.800 ptas.; \$ USA 26.00.

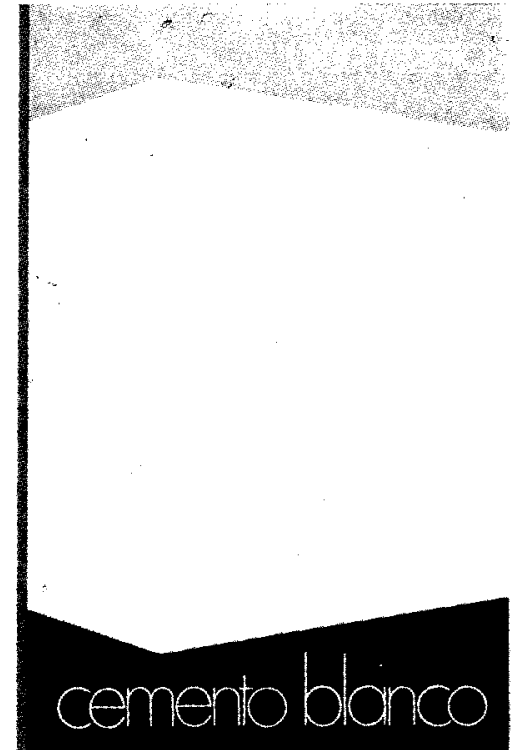

\section{Cemento blanco}

Julián Rezola

Ingeniero Químico Dipl. I. O.S

Sabido es que existe una extensa y documentada bibliografia sobre el cemento gris: en cambio, no puede decirse lo mismo acerca del cemento portiand blanco, ya que los escritos existentes se refieren tan sólo a algunas peculiaridades que le distinguen de aquét

El autor nos ofrece sus profundos conocimientos y su larga experiencia tanto en laboratorio como en fabricación.

La parte descriptiva del libro se complementa con gráficos, diagramas $\mathrm{y}$ fotografias de gran utilidad destinados a conseguir la aplicación apropiada de este aglomerante.

Un volumen encuadernado en cartoné policerado, de $17,4 \times 24,3 \mathrm{~cm}$, compuesto de 395 páginas,
numerosas figuras, tablas y ábacos.

Precios: España, 1.700 ptas.; extranjero, $\$ 34$.

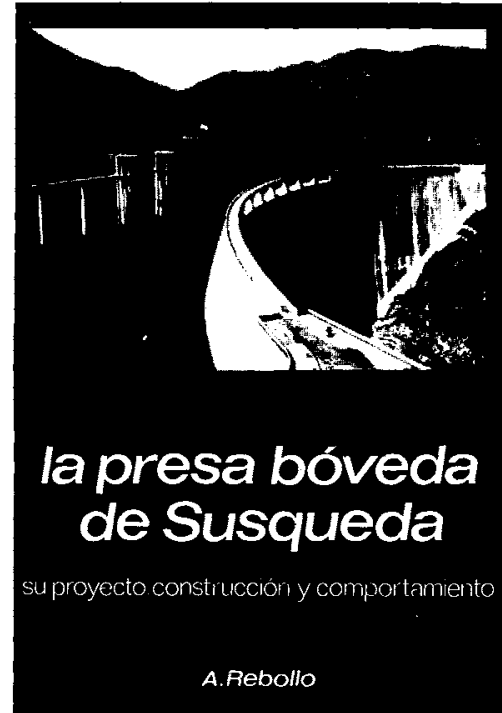

La presa bóveda de Susqueda

A. Rebollo,

Dr. Ingeniero de Caminos

El esfuerzo del constructor de presas se sitúa por su pretensión de perennidad, a contracorriente de las pendencias de la cividizacion actual, caracterizada por lo tus de la civizacion actua car 10.000 que están envejeciendo $y$ reclaman construcción gerontológicos para mantener y perfeccionar servicio y garantizar su inalienable pretensión de perennidad. En la medida en que todas nuevas obras, grandes o pequeñas, son portadoras de riesgos ecológicos $y_{1}$ a veces, catastróficos, que aumentan con el envejecimiento, la gerontologia de las presas es todo un emplazo. La acción adelantada de Arturo Rebollo en este terreno marca un camino a seguir para todos los que aman su propia obra con la devoción paternal que él ha puesto en Susqueda.

Un volumen encuadernado en cartone plastificado con tomo de tela, de $18 \times 24,5 \mathrm{~cm}$, compuesto de 408 páginas, 330 figuras y fotografias y 39 tablas. Precios: 1.700 ptas.; extranjero, \$ USA 24.00. 